\title{
Investigation of guided wave properties of anisotropic composite laminates using a semi-analytical finite element method
}

\author{
Wenbo Duan; Tat-Hean Gan \\ Brunel Innovation Centre, Brunel University London, Uxbridge, Middlesex, UK, UB8 3PH
}

Abstract: Composite materials have been widely used in various applications, and guided waves are often used as a non-destructive testing tool to inspect defects or damages in composite laminates. A composite laminate is an assembly of many composite layers with anisotropic material properties and an arbitrary fibre orientation in each layer. Guided wave properties of Lamb and shear-horizontal waves are thus direction dependent. As a result, the group velocity of each wave mode has a component parallel to the wave propagation direction, and also a component perpendicular to the wave propagation direction. In this article, a semi-analytical finite element method is developed to model composite laminates with an arbitrary fibre orientation and also anisotropic material properties in each layer. The Galerkin's principle is used to derive the weak forms of the governing equations, and an energy velocity formulation is used to derive the parallel and perpendicular energy velocities. The finite element solutions are compared against available analytical and numerical solutions in the literature for forward waves, and excellent agreements have been achieved. On this basis, guided wave properties of backward waves are investigated. It is well understood that in an isotropic plate, the energy velocity of a backward wave is directly opposite to the phase velocity, i.e., $180^{\circ}$ out of phase. However, in a composite laminate, the energy velocity of a backward wave is normally not exactly $180^{\circ}$ out of phase with the phase velocity due to the existence of a skew angle. The angular dependences of wave properties of forward and backward waves are investigated in this article. 


\section{Introduction}

Composite materials are widely used because of their lighter and stronger properties. Guided waves are often used to inspect defects or damages in composite laminates. Composite laminates have anisotropic material properties, and wave propagation properties are thus frequency and direction dependent. Furthermore, group velocities have components parallel and perpendicular to the wave propagation vector direction, and group velocity wave front curves are thus complicated. Guided wave properties are well studied for forward waves, however, backward waves in composite laminates haven't been presented or discussed in the literature. In this article, a Galerkin based semi-analytical finite element (SAFE) method is derived to study guided wave properties of forward and backward Lamb and shear-horizontal waves. This requires only the thickness of composite laminates to be discretised. An energy velocity formation is used to calculate energy velocities in two orthogonal directions, which avoids the need to calculate wavenumber derivatives through a finite difference scheme. Guided wave properties of forward and backward waves are presented and compared to available analytical and numerical solutions in the literature.

In a classical article, Lamb originally derived exact analytical governing equations for wave propagation in an isotropic two dimensional layer, and these waves were later named after him [1]. However, these governing equations couldn't be solved analytically, and Lamb only presented solutions related to long and short wavelength limits [1]. The full numerical solutions to these equations were presented by Mindlin four decades later [2]. In addition to forward waves, backward waves have been observed [2,3]. These waves have opposite phase and group velocities. Backward waves form small loops with group velocities both starting and ending at zero. Phase velocities start from a finite value and increase to infinity 
in the backward wave frequency regime. Negishi reported that the first backward wave exists only when Poisson's ratio of the isotropic plate is less than 0.45 , and the second backward wave exists when Poisson's ratio is less than 0.31 [4]. Backward waves include zero group velocity points which are associated with resonance and ringing effects. Transmitted sound energy will be localised at these points, and this phenomenon could be important in nondestructive testing, material characterisation applications etc [5-15].

Analytical and numerical approaches have been used to investigate backward waves in a layered medium with isotropic material properties in each layer $[16,17]$. The continuous material variation through thickness could be represented by a number of numerically discretised finite element layers [17]. Existence of backward waves was also found in immersed layered cylindrical shells with isotropic material properties [18]. Solie and Auld proposed a displacement partial wave technique to study dispersion relations for elastic waves in a single layer plate of arbitrary anisotropy [19]. This principle has been used to study wave propagation in anisotropic plates with different groups of material properties [2022]. Hussain and Ahmad studied locations of zero-group velocity points for a single layer orthotropic plate [23]. Analytical governing equations were derived for wavenumber solutions of symmetric and antisymmetric modes. Laurent et al used a displacement potential technique to calculate dispersion properties of backward waves in a transversely isotropic cylinder [24]. Shuvalov and Poncelet presented a leading-order dispersion coefficient method to identify backward wave branches near thickness resonances, without the need to calculate the full dispersion curves [25]. This applies to a single layer plate of arbitrary anisotropy.

However, composite laminates include not only anisotropic material properties, but also multiple layers with a different fibre orientation in each layer. The calculation of dispersion 
properties in composite laminates is thus complicated. The transfer or global matrix technique could be used to study wave propagation in layered composite plates [26-33]. The transfer matrix defines displacements and stresses at the top of a layer with respect to those at the bottom of the layer. The recurrence formula and continuity conditions at interfaces enabled quantities at the $n$th layer to be expressed in terms of corresponding quantities at the first layer. The global matrix technique assembles all the equations for all the layers, instead of multiplying all the transfer matrices in a recursive way. While these articles discussed the techniques to solve the governing equations, the physical properties of guided waves in composite laminates were not fully exploited. Wang and Yuan investigated wave properties of symmetric composite laminates both theoretically and experimentally in detail [34]. In particular, group velocity wave front curves were analysed, and the skew angle between phase and group velocity vectors was discussed, due to anisotropic material properties. Karmazin et al presented the Green's matrix technique to study wave propagation in anisotropic multi-layered composites [35]. An algorithm of constructing the Fourier transform of the Green's matrix of the given boundary problem was presented. Ma et al adopted the reverberation-ray matrix method to study dispersion properties of Lamb waves in composite laminates [36]. Thierry et al proposed the Wave Finite Element Method to study dispersion properties of guided waves in two-dimensional periodic textile composites, and the Component Mode Synthesis method was further used to reduce the size of the stiffness matrix [37]. Liu et al studied the frequency response of displacement and velocity to external sources in a two dimensional isotropic or cross-ply composite plate [38]. It was found that the displacement frequency response amplitude is very large within or near the backward wave region. Gravenkamp et al used the Scaled Boundary Finite Element Method (SBFEM) to compute dispersion properties of guided waves in plate structures with isotropic or functionally graded material properties [39]. 
In this article, a semi-analytical finite element method is developed to study dispersion properties of guided waves in composite laminates with anisotropic material properties in each layer. A weighted residual method based on Galerkin's principle is used to derive the weak form equations. Numerical solutions of the final eigen equation deliver both forward and backward waves. An energy velocity based formulation is used to separate backward waves from forward waves. In addition, slowness curves and group velocity wave front curves are presented for typical forward and backward waves in symmetric and nonsymmetric composite laminates. The paper is organised as follows. Section 2 describes the development of the semi-analytical finite element equations for composite laminates with anisotropic material properties and an arbitrary fibre orientation in each layer. In section 3 , the finite element solutions are compared to available analytical and numerical solutions for forward waves in a symmetric composite laminate, and excellent agreements have been achieved. Guided wave properties are then presented for backward waves. In section 4, modal properties for forward and backward waves in a non-symmetric composite laminate are presented, and conclusions are drawn in section 5.

2. Semi-analytical finite element method for composite laminates

In this paper, it is assumed that a composite laminate includes $m$ layers with an arbitrary fibre orientation in each layer. The fibre orientation in the $j$ th lamina is shown in Fig. 1 with principle material $x_{j}^{\prime}-y_{j}^{\prime}-z_{j}^{\prime}$ directions and global $x-y-z$ directions. $z$ direction is defined normal to the lamina surface, and $z_{j}^{\prime}$ is parallel to $z$. The global $x$ is defined parallel to the wave propagation direction in the composite laminate. This facilitates the development of equations. Here, the local principle $x_{j}^{\prime}-y_{j}^{\prime}$ plane could be rotated about the $z_{j}^{\prime}$ axis to global $x$ $y$ plane. The rotation angle from the local coordinate system to the global coordinate system is $\theta_{j}$, shown in Fig. 1 . 


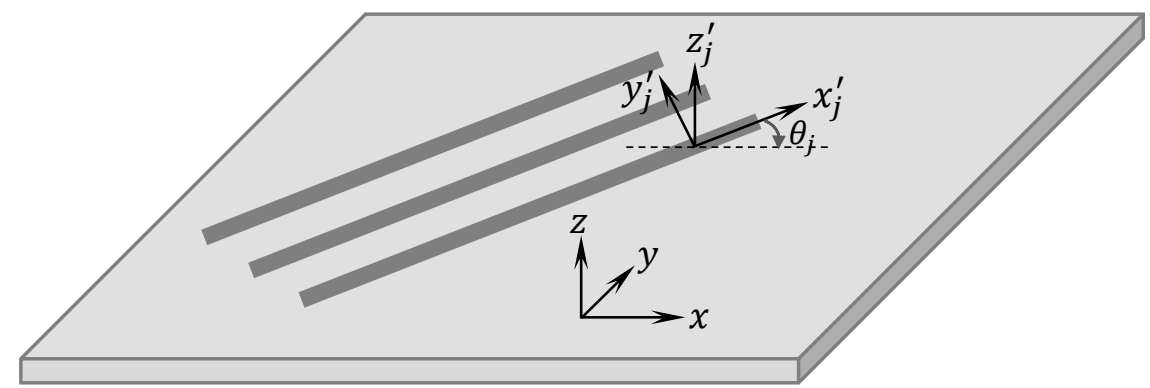

Fig. 1 Fibre orientation in the $j$ th lamina with principle material $x_{j}^{\prime}-y_{j}^{\prime}-z_{j}^{\prime}$ directions and global $x-y-z$ directions.

The governing equations for wave propagation in the $j$ th lamina are defined as $(j=1$ to $m$ ) [30]:

$$
\begin{aligned}
\rho_{j} \frac{\partial^{2} u_{x_{j}}^{*}}{\partial t^{2}} & =\frac{\partial \sigma_{x x_{j}}^{*}}{\partial x}+\frac{\partial \sigma_{x y_{j}}^{*}}{\partial y}+\frac{\partial \sigma_{x z_{j}}^{*}}{\partial z} \\
\rho_{j} \frac{\partial^{2} u_{y_{j}}^{*}}{\partial t^{2}} & =\frac{\partial \sigma_{y x_{j}}^{*}}{\partial x}+\frac{\partial \sigma_{y y_{j}}^{*}}{\partial y}+\frac{\partial \sigma_{y z_{j}}^{*}}{\partial z} \\
\rho_{j} \frac{\partial^{2} u_{z_{j}}^{*}}{\partial t^{2}} & =\frac{\partial \sigma_{z x_{j}}^{*}}{\partial x}+\frac{\partial \sigma_{z y_{j}}^{*}}{\partial y}+\frac{\partial \sigma_{z z_{j}}^{*}}{\partial z}
\end{aligned}
$$

Here, $\rho_{j}$ is density, $t$ is time, $u^{*}$ is displacement and $\sigma_{q l}^{*}(q, l=x, y$ or $z)$ is a symmetric stress tensor of rank two. A time dependence of $e^{i \omega t}$ is assumed throughout this article, where $\omega$ is the radian frequency and $i=\sqrt{-1}$.

The displacement $u^{*}$ in the composite laminate can be expressed in the form

$$
u_{q}^{*}(z)=u_{q}(z) e^{i(\omega t-k x)}
$$


where $u_{q}$ is an eigenfunction $(q=x, y$ or $z)$.

The weighted residual method is used and Eqs. (1a)-(1c) are then weighted using an arbitrary weighting function $w_{q}$. This procedure has been discussed in detail by Duan et al for isotropic fluid and elastic materials [40-43]. Substituting Eq. (2) into Eqs. (1a)-(1c) and enforcing continuity of displacement and normal stresses over the interfaces between adjacent layers, the weak form governing equations could be obtained through integration by parts:

$$
\begin{gathered}
\int_{\mathrm{z}=h_{0}}^{\mathrm{z}=h_{m}}\left(\frac{\partial w_{q}}{\partial y} \sigma_{q y}+\frac{\partial w_{q}}{\partial z} \sigma_{q z}\right) d \mathrm{z}+\int_{\mathrm{z}=h_{0}}^{\mathrm{z}=h_{m}} w_{q}\left\{i k \sigma_{q x}-\rho \omega^{2} u_{q}\right\} d \mathrm{z} \\
=\left.w_{q}\left(\sigma_{q z} n_{z}\right)\right|_{\mathrm{z}=h_{m}}-\left.w_{q}\left(\sigma_{q z} n_{z}\right)\right|_{\mathrm{z}=h_{0}}, \quad q=x, y \text { or } z
\end{gathered}
$$

Here, the integration is carried out in the $z$ direction and covers all the layers in the composite laminate. $h_{0}$ is the $\mathrm{z}$ axis position at the bottom of the laminate, and $h_{m}$ is the $\mathrm{z}$ axis position at the top of the laminate. Note that any integration on interfaces between adjacent layers would be cancelled out, because surface normals are opposite to each other between adjacent layers.

Composite materials have orthotropic material properties in the principle $x_{j}^{\prime}-y_{j}^{\prime}-z_{j}^{\prime}$ coordinates in each layer. In the transformed global $x-y-z$ coordinates, the material properties would be monoclinic symmetric having $x-y$ as a plane of symmetry. The stress-strain relations in the global coordinates are given as:

$$
\left\{\begin{array}{l}
\sigma_{x x} \\
\sigma_{y y} \\
\sigma_{z z} \\
\sigma_{y z} \\
\sigma_{x z} \\
\sigma_{x y}
\end{array}\right\}=\left[\begin{array}{cccccc}
C_{11} & C_{12} & C_{13} & 0 & 0 & C_{16} \\
C_{12} & C_{22} & C_{23} & 0 & 0 & C_{26} \\
C_{13} & C_{23} & C_{33} & 0 & 0 & C_{36} \\
0 & 0 & 0 & C_{44} & C_{45} & 0 \\
0 & 0 & 0 & C_{45} & C_{55} & 0 \\
C_{16} & C_{26} & C_{36} & 0 & 0 & C_{66}
\end{array}\right]\left\{\begin{array}{c}
\varepsilon_{x x} \\
\varepsilon_{y y} \\
\varepsilon_{z z} \\
2 \varepsilon_{y z} \\
2 \varepsilon_{x z} \\
2 \varepsilon_{x y}
\end{array}\right\}
$$


The stiffness matrix in Eq. (4) is layer and fibre orientation dependent. For the clarity of the presentation, the subscript $j$ is ignored in Eq. (4). The relations between stiffness constants in the principle local coordinates and in the global coordinates as a function of rotation angle $\theta_{j}$ are given in Appendix A. In engineering applications, the material properties for composite materials are normally defined as engineering constants, i.e., Young's modulus, Poisson's ratio etc. The relations between principle stiffness constants and engineering constants are given in Appendix B.

The strain-displacement relations are $(q, l=x, y$ or $z)$ :

$$
\varepsilon_{q l}=\frac{1}{2}\left(\frac{\partial u_{l}}{\partial x_{q}}+\frac{\partial u_{q}}{\partial x_{l}}\right)
$$

The displacement is discretised following the conventional finite element procedure $(q=$ $x, y$ or $z)$ :

$$
u_{q}(z)=\mathbf{N}_{\mathrm{q}} \mathbf{u}_{\mathrm{q}}
$$

Here, $\mathbf{N}_{\mathrm{q}}$ is the global shape function in the form of a row vector, and $\mathbf{u}_{\mathrm{q}}$ is the nodal position displacement in the form of a column vector.

Now substitute Eqs. (4-6) into Eq. (3) and set the weighting functions equal to the shape functions. The governing equations can be assembled in the matrix form as:

$$
\left[\begin{array}{cccccc}
Z_{1 x} & Z_{1 y} & 0 & 0 & 0 & R_{1 z} \\
Z_{1 y}^{T} & Z_{2 y} & 0 & 0 & 0 & R_{2 z} \\
0 & 0 & Z_{3 z} & -R_{1 z}^{T} & -R_{2 z}^{T} & 0 \\
0 & 0 & 0 & 1 & 0 & 0 \\
0 & 0 & 0 & 0 & 1 & 0 \\
0 & 0 & 0 & 0 & 0 & 1
\end{array}\right]\left\{\begin{array}{c}
u_{x} \\
u_{y} \\
u_{z} \\
i k u_{x} \\
i k u_{y} \\
i k u_{z}
\end{array}\right\}
$$




$$
=i k\left[\begin{array}{cccccc}
0 & 0 & 0 & T_{1 x} & T_{1 y} & 0 \\
0 & 0 & 0 & T_{1 y}^{T} & T_{2 y} & 0 \\
0 & 0 & 0 & 0 & 0 & T_{3 z} \\
1 & 0 & 0 & 0 & 0 & 0 \\
0 & 1 & 0 & 0 & 0 & 0 \\
0 & 0 & 1 & 0 & 0 & 0
\end{array}\right]\left\{\begin{array}{c}
u_{x} \\
u_{y} \\
u_{z} \\
i k u_{x} \\
i k u_{y} \\
i k u_{z}
\end{array}\right\}
$$

where

$$
\begin{gathered}
Z_{1 x}=\int_{\mathrm{z}=h_{0}}^{\mathrm{z}=h_{m}}\left[C_{55} \frac{\partial \mathbf{N}^{\mathrm{T}}}{\partial z} \frac{\partial \mathbf{N}}{\partial z}-\rho \omega^{2} \mathbf{N}^{\mathrm{T}} \mathbf{N}\right] d \mathrm{z} \\
Z_{1 y}=\int_{\mathrm{z}=h_{0}}^{\mathrm{z}=h_{m}}\left[C_{45} \frac{\partial \mathbf{N}^{\mathrm{T}}}{\partial z} \frac{\partial \mathbf{N}}{\partial z}\right] d \mathrm{z} \\
R_{1 z}=\int_{\mathrm{z}=h_{0}}^{\mathrm{z}=h_{m}}\left[-C_{55} \frac{\partial N_{i}}{\partial z} N_{j}+C_{13} N_{i} \frac{\partial N_{j}}{\partial z}\right] d \mathrm{z} \\
R_{2 z}=\int_{\mathrm{z}=h_{0}}^{\mathrm{z}=h_{m}}\left[-C_{45} \frac{\partial N_{i}}{\partial z} N_{j}+C_{36} N_{i} \frac{\partial N_{j}}{\partial z}\right] d \mathrm{z} \\
T_{1 x}=\int_{\mathrm{z}=h_{0}}^{\mathrm{z}=h_{m}}\left[C_{11} N_{i} N_{j}\right] d \mathrm{z} \\
Z_{2 y}=\int_{\mathrm{z}=h_{0}}^{\mathrm{z}=h_{m}}\left[C_{44} \frac{\partial N_{i}}{\partial z} \frac{\partial N_{j}}{\partial z}-\rho \omega^{2} N_{i} N_{j}\right] d \mathrm{z} \\
\left.T_{1 y}=\int_{\mathrm{z}=h_{0}}^{\mathrm{z}=h_{m}}\left[C_{66} N_{i} N_{j}\right] d \mathrm{z} N_{i} N_{j}\right] d \mathrm{z}
\end{gathered}
$$




$$
\begin{gathered}
Z_{3 z}=\int_{\mathrm{z}=h_{0}}^{\mathrm{z}=h_{m}}\left[C_{33} \frac{\partial N_{i}}{\partial z} \frac{\partial N_{j}}{\partial z}-\rho \omega^{2} N_{i} N_{j}\right] d \mathrm{z} \\
T_{3 z}=\int_{\mathrm{z}=h_{0}}^{\mathrm{z}=h_{m}}\left[C_{55} N_{i} N_{j}\right] d \mathrm{z}
\end{gathered}
$$

In Eqs. (8)-(17), the stiffness constants are layer dependent. The solution of Eq. (7) delivers $6 N_{p}$ eigenmodes, where $N_{p}$ is the total number of nodes in the composite laminate. These modes include propagating and evanescent modes. To separate and group modes at adjacent frequencies automatically, a modal assurance criterion (MAC) is used [44]:

$$
M A C=\frac{\left|\int_{\mathrm{z}=h_{0}}^{\mathrm{z}=h_{m}} \sum_{q=x, y, z}\left[u_{q}\left(f_{1}\right) * u_{q}^{T}\left(f_{2}\right)\right] d \mathrm{z}\right|^{2}}{\int_{\mathrm{z}=h_{0}}^{\mathrm{z}=h_{m}} \sum_{q=x, y, z}\left[u_{q}\left(f_{1}\right) * u_{q}^{T}\left(f_{1}\right)\right] d \mathrm{z} \cdot \int_{\mathrm{z}=h_{0}}^{\mathrm{z}=h_{m}} \sum_{q=x, y, z}\left[u_{q}\left(f_{2}\right) * u_{q}^{T}\left(f_{2}\right)\right] d \mathrm{z}}
$$

Here, $u_{q}\left(f_{1}\right)$ and $u_{q}\left(f_{2}\right)$ are modal displacements at adjacent frequencies $f_{1}$ and $f_{2}$ respectively, and the superscript $T$ is a conjugate operator. The MAC value in Eq. (18) indicates the consistence (degree of linearity) of any two modes, and the same mode would have a high consistence value at two adjacent frequencies. Evaluation of the MAC values for all the propagating modes at all the frequencies would allow modes to be grouped and sorted automatically.

The calculation of the phase velocity follows the usual definition, i.e., $c_{p}=\omega / k$. In the current coordinate system, the phase velocity is parallel to the wave propagation direction, which is the $x$ direction. Clearly, the phase velocity is propagation angle dependent in a composite laminate. The group velocity has two components, one in the wave propagation 
direction, and one perpendicular to the wave propagation direction, due to anisotropic material properties. Wang and Yuan proposed a finite difference scheme to calculate group velocities at two orthogonal directions [34]. In this article, an alternative energy velocity based formulation is used to calculate energy velocity at each frequency, which is equal to the group velocity for a lossless system [45]. The energy velocity components at two perpendicular directions are defined as:

$$
c_{e q}=\frac{\int_{\mathrm{z}=h_{0}}^{\mathrm{z}=h_{m}} \sum_{l=x, y, z} \operatorname{real}\left[\sigma_{q l} *\left(i \omega u_{l}^{T}\right)\right] d \mathrm{z}}{\int_{\mathrm{z}=h_{0}}^{\mathrm{z}=h_{m}} \rho \omega^{2} \sum_{l=x, y, z} \operatorname{real}\left[u_{l} * u_{l}^{T}\right] d \mathrm{z}}, \quad q=x \text { or } y
$$

Here, $c_{e x}$ is the energy velocity component parallel to the wave propagation direction (wave number direction), and $c_{e y}$ is the energy velocity component perpendicular to the wave propagation direction. In an isotropic plate, modal properties are the same at all directions, and thus $c_{e y}$ is zero. However, in a composite laminate, $c_{e y}$ is normally not zero. The magnitude of the energy velocity and the skew angle are given by:

$$
c_{e}=\sqrt{\left(c_{e x}\right)^{2}+\left(c_{e y}\right)^{2}}, \quad \text { and } \theta_{s}=\tan ^{-1} \frac{c_{e y}}{c_{e x}}
$$

The presence of backward waves can be determined by the sign of $c_{e x}$ calculated from Eq. (19). For an isotropic plate, $c_{e x}$ is positive for forward waves, and negative for backward waves (opposite phase and group velocities). The same sign indication can be used for determination of forward and backward waves in composite laminates. However, the overall energy velocity magnitude $c_{e}$ defined in Eq. (20) can only be positive. It should be noted that the energy velocity is normally not in phase or out of phase with the phase velocity in composite laminates. A schematic view of the relationship between the wave propagation vector and the energy velocity vector is shown in Fig. 2 for both forward and backward waves. For a conventional forward wave, the energy velocity and the phase velocity is roughly pointing in the same direction, with a skew angle in the vicinity of zero. For a 
backward wave, the energy velocity and the phase velocity is pointing in opposite directions, with a skew angle in the vicinity of $\pi$.

(a)

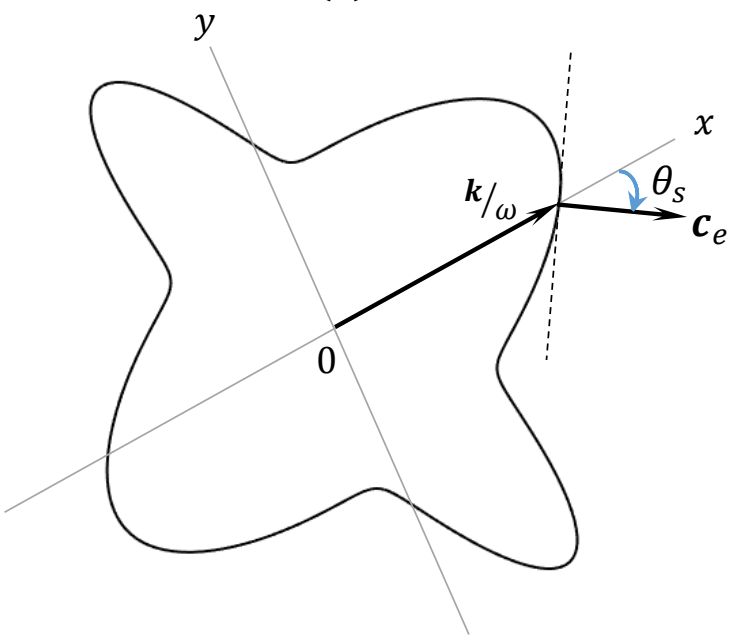

(b)

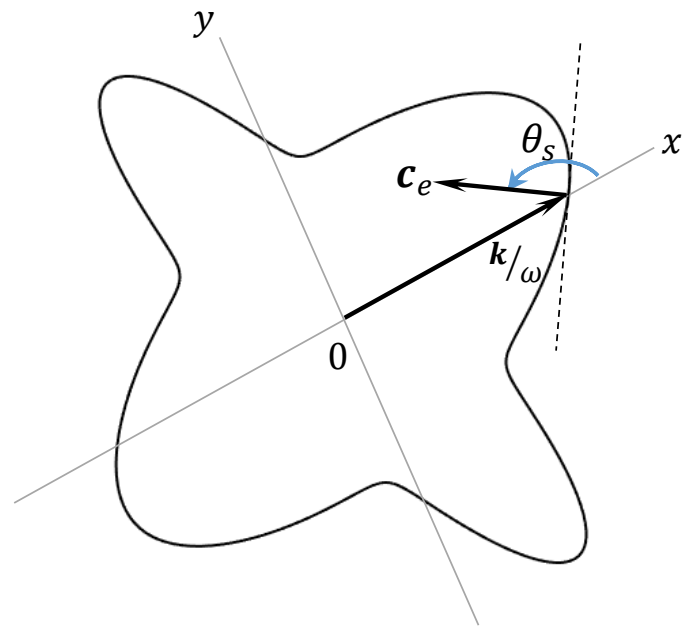

Fig. 2 Schematic relationship between the propgation vector and the energy velocity vector: (a) forward wave; (b) backward wave.

3. Numerical solutions for a symmetric laminate

The SAFE model developed in section 2 is programmed in Matlab, and the numerical solutions are presented here. The symmetric laminate investigated by Wang and Yuan [34] is chosen to validate the SAFE model. This example has also been studied by Karmazin et al [35] and Ma et al [36], using different approaches. In this section, modal properties of both forward and backward waves are presented. The material properties of an AS4/3502 composite lamina are given in Table 1 . These are material properties defined in the local coordinate system. 
Table 1. Material properties of an AS4/3502 composite lamina (units: Gpa, $\mathrm{kg} / \mathrm{m}^{3}$ )

\begin{tabular}{|c|c|c|c|c|c|c|c|c|c|}
\hline$E_{1}^{\prime}$ & $E_{2}^{\prime}$ & $E_{3}^{\prime}$ & $G_{12}^{\prime}$ & $G_{13}^{\prime}$ & $G_{23}^{\prime}$ & $v_{12}^{\prime}$ & $v_{13}^{\prime}$ & $v_{23}^{\prime}$ & $\rho$ \\
\hline 127.6 & 11.3 & 11.3 & 5.97 & 5.97 & 3.75 & 0.3 & 0.3 & 0.34 & 1578 \\
\hline
\end{tabular}

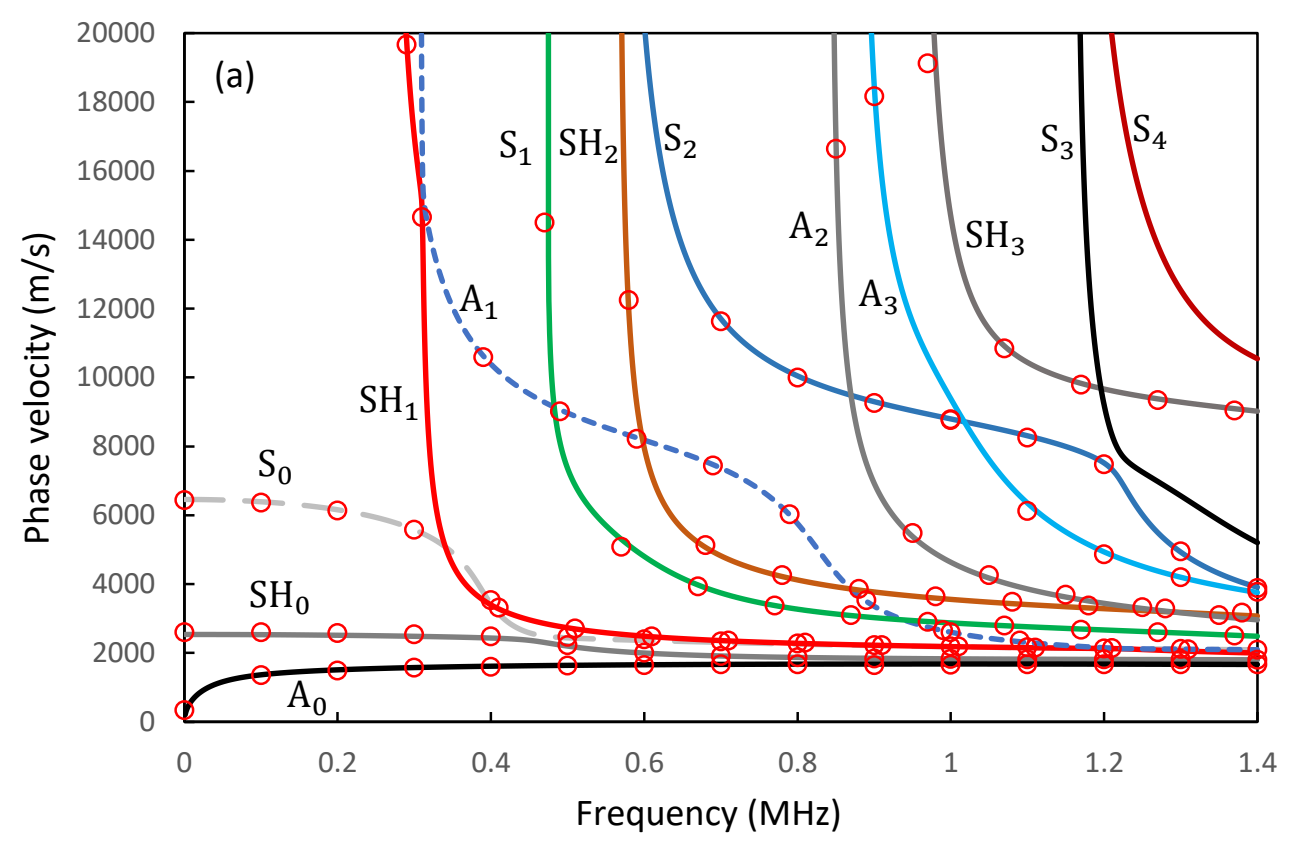



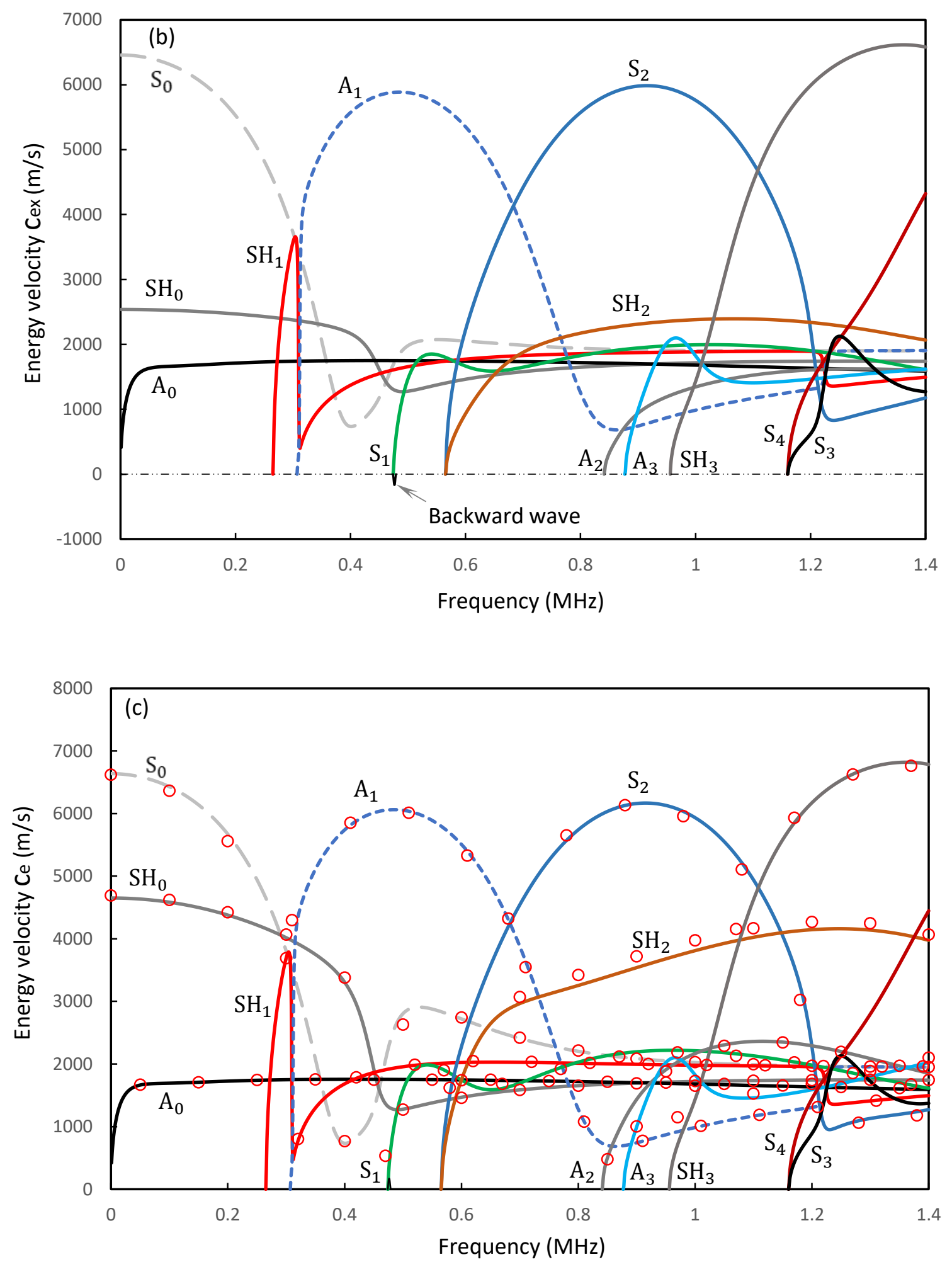

Fig. 3. Dispersion curves for guided waves propagating alone $30^{\circ}$ direction in a $\left[+45_{6} /-45_{6}\right]_{s}$ laminate: (a) phase velocity; (b) energy velocity component $c_{e x}$; (c) energy velocity $c_{e}$. - : current numerical solution; o o 0 : the transfer matrix solution [34]. 
Fig. 3 presents phase and energy velocity dispersion curves for guided waves propagating alone $30^{\circ}$ direction in a $\left[+45_{6} /-45_{6}\right]_{s}$ laminate. This is a symmetric laminate with 24 plies. The total thickness of the laminate is $h=3 \mathrm{~mm}$. The modes are labelled according to their dominant structure of the displacements in a representative frequency range [34], i.e., symmetric (S mode), anti-symmetric (A mode) or shear-horizontal (SH mode). Furthermore, all the modes have displacements in $x, y$ and $z$ directions in composite laminates. Figs. 3(a) and (c) show excellent agreement between the current numerical solution and the transfer matrix solution of Wang and Yuan [34]. In principle, energy velocity equals group velocity for the lossless system studied here. Fig. 3(b) shows the energy velocity component in the wave propagation direction, i.e., $c_{e x}$. The presence of a backward wave is clearly visible, with a negative energy velocity. This mode spans in a frequency range less than $4 \mathrm{kHz}$, and could thus be easily overlooked. The characteristics of this mode are further examined later on in this section.

Fig. 4 presents slowness curves of guided waves in the $\left[+45_{6} /-45_{6}\right]_{s}$ laminate at a nondimensional frequency of $\omega h / c_{s}=4$. Here, $h$ is the thickness of the laminate, and $c_{s}$ is the shear velocity defined by $c_{S}=\sqrt{G_{12}^{\prime} / \rho}$. Slowness is used to define the time required for a mode to travel a unit distance, i.e., $S=k / \omega$. Slowness is direction dependent, and the slowness vector direction is the same as the phase velocity direction. Fig. 4 shows that the current numerical solution overlays the transfer matrix solution [34] at all the propagation directions. Fig. 5 shows the energy velocity wave front curves in the $\left[+45_{6} /-45_{6}\right]_{s}$ laminate at $\omega h / c_{s}=4$. The current numerical solution agrees very well with the Green's matrix solution [35]. Note that the energy velocity wave vector is not parallel to the wave propagation vector, which has been shown in Fig. 2. The skew angles have to be taken into consideration when plotting energy velocity wave front curves. It is thus possible for several wave propagation directions to be responsible for the same energy velocity direction, which 
has been shown in Fig. 5 for shear-horizontal wave modes $\mathrm{SH}_{0}$ and $\mathrm{SH}_{1}$. This would then produce energy focusing. Figs. 4 and 5 also show that the fundamental anti-symmetric mode $\mathrm{A}_{0}$ is less fibre orientation dependent. The slowness and energy velocity wave front curves of this mode are close to a circle, which indicates a quasi-isotropic behaviour for this particular laminate. All the other modes exhibit strong anisotropic behaviour.

(a)

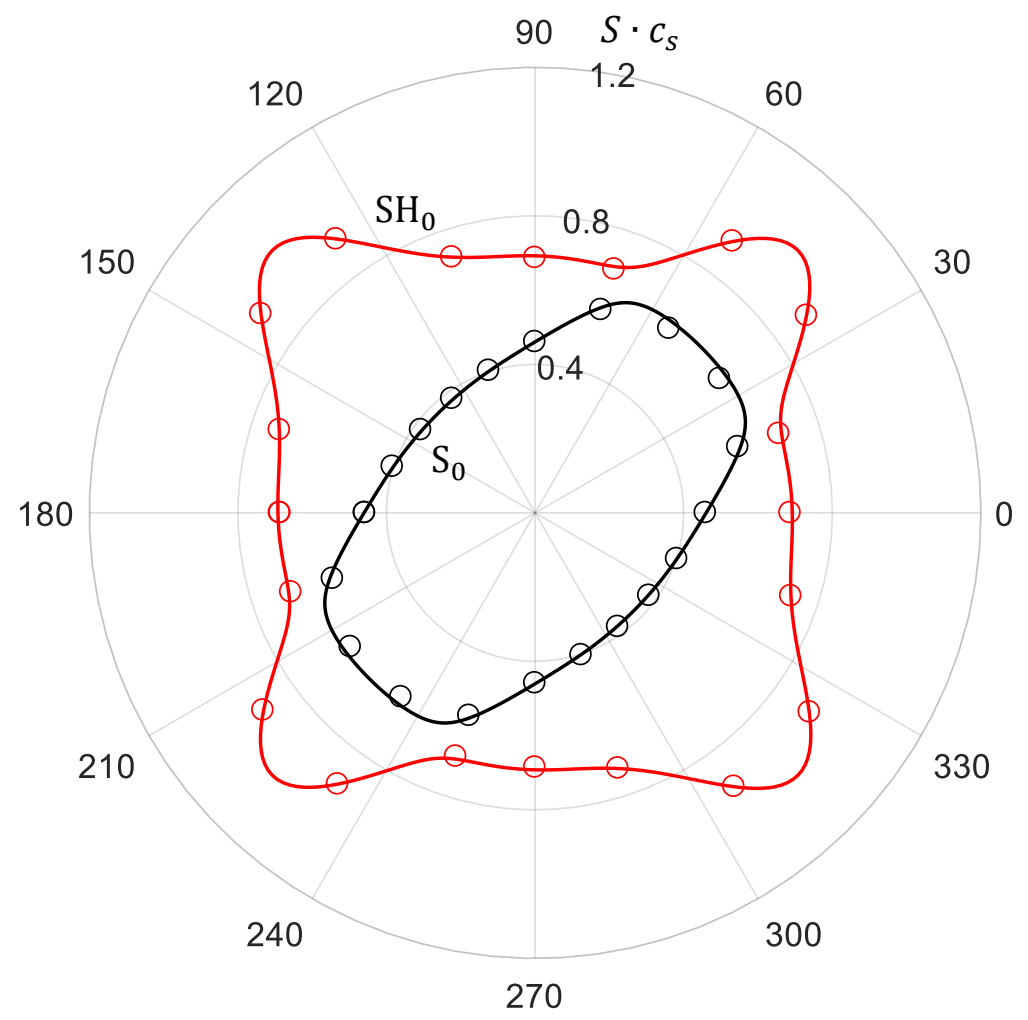


(b)

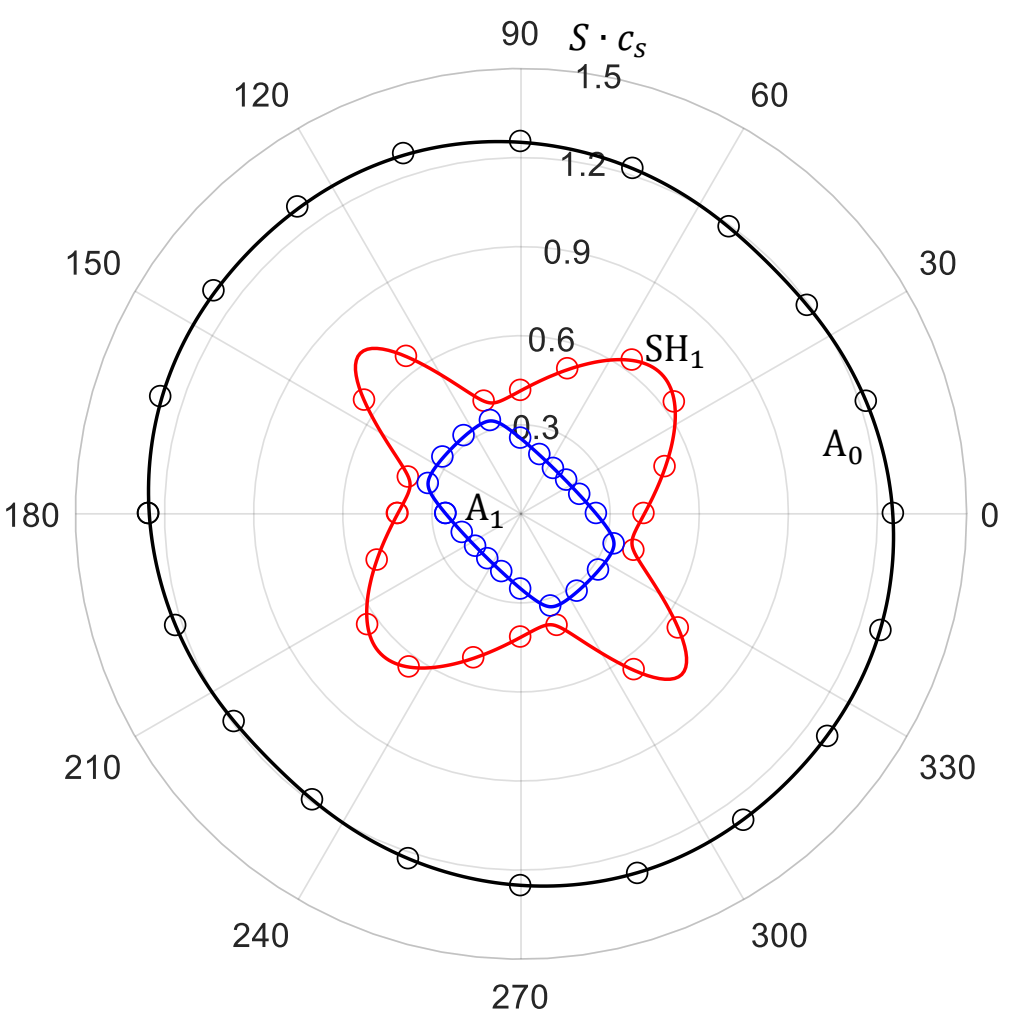

Fig. 4 Slowness curves of guided waves in the $\left[+45_{6} /-45_{6}\right]_{s}$ laminate at $\omega h / c_{s}=4$ : (a) symmetric modes; (b) anti-symmetric modes. ——: current numerical solution; o o o: the transfer matrix solution [34]. 
(a)

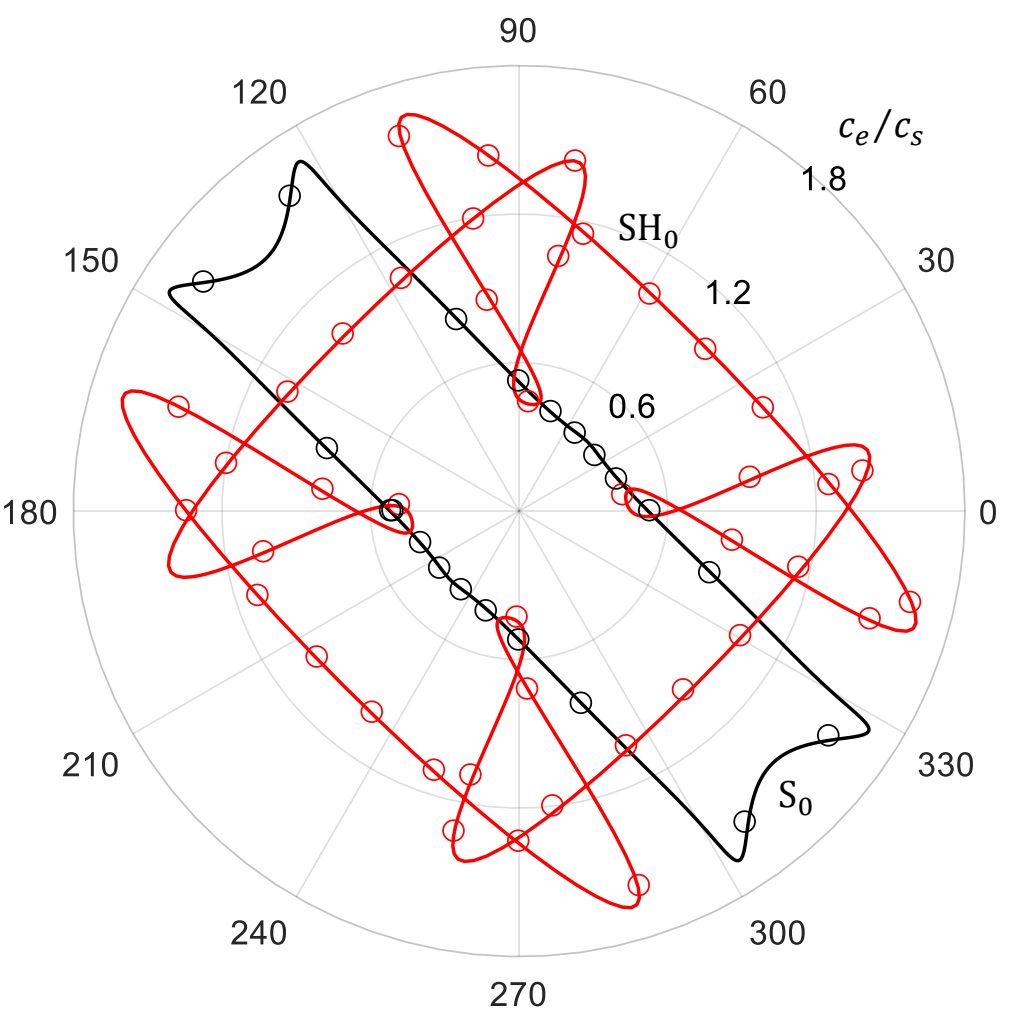

(b)

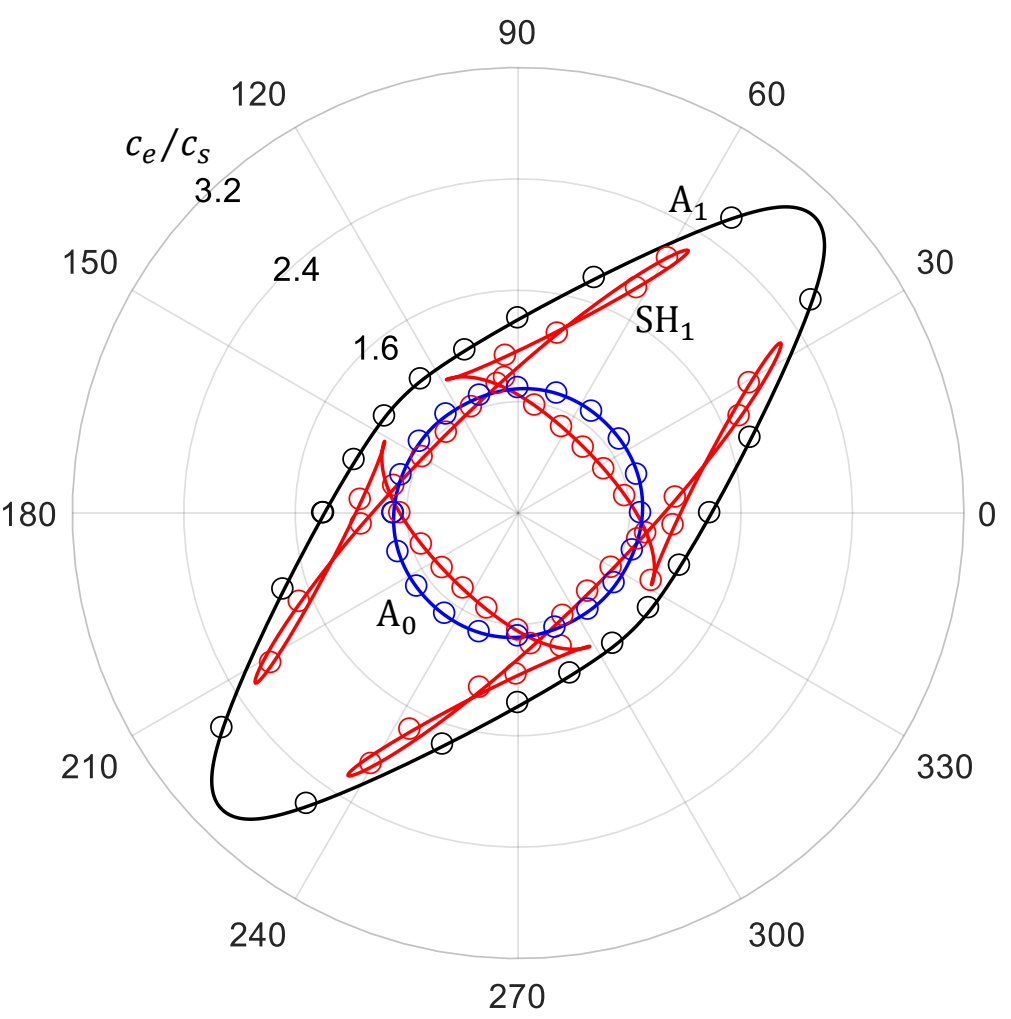


Fig. 5 Energy velocity wave front curves of guided waves in the $\left[+45_{6} /-45_{6}\right]_{s}$ laminate at $\omega h / c_{s}=4:$ (a) symmetric modes; (b) anti-symmetric modes. : current numerical solution; o o o: the Green's matrix solution [35].

To further examine properties of the backward wave, dispersion curves around the backward wave region have been shown in Fig. 6. The backward wave is a symmetric wave, cutting on in association with the symmetric Lamb mode S1. Phase velocity of the backward wave starts from a finite value, and increases to infinity in the backward wave region. The energy velocity component $c_{e x}$ starts and ends at zero, forming a small loop. This is similar to conventional backward waves in an isotropic plate. However, Fig. 6(c) shows that the energy velocity magnitude $c_{e}$ starts from a finite value and ends at zero. This difference is coming from the energy velocity component in the direction perpendicular to the wave propagation direction. The zero group velocity point (with resonance effects) is associated with the start of the backward wave region. At this frequency, the phase velocity is finite, and the energy velocity component $c_{e x}$ is zero. 

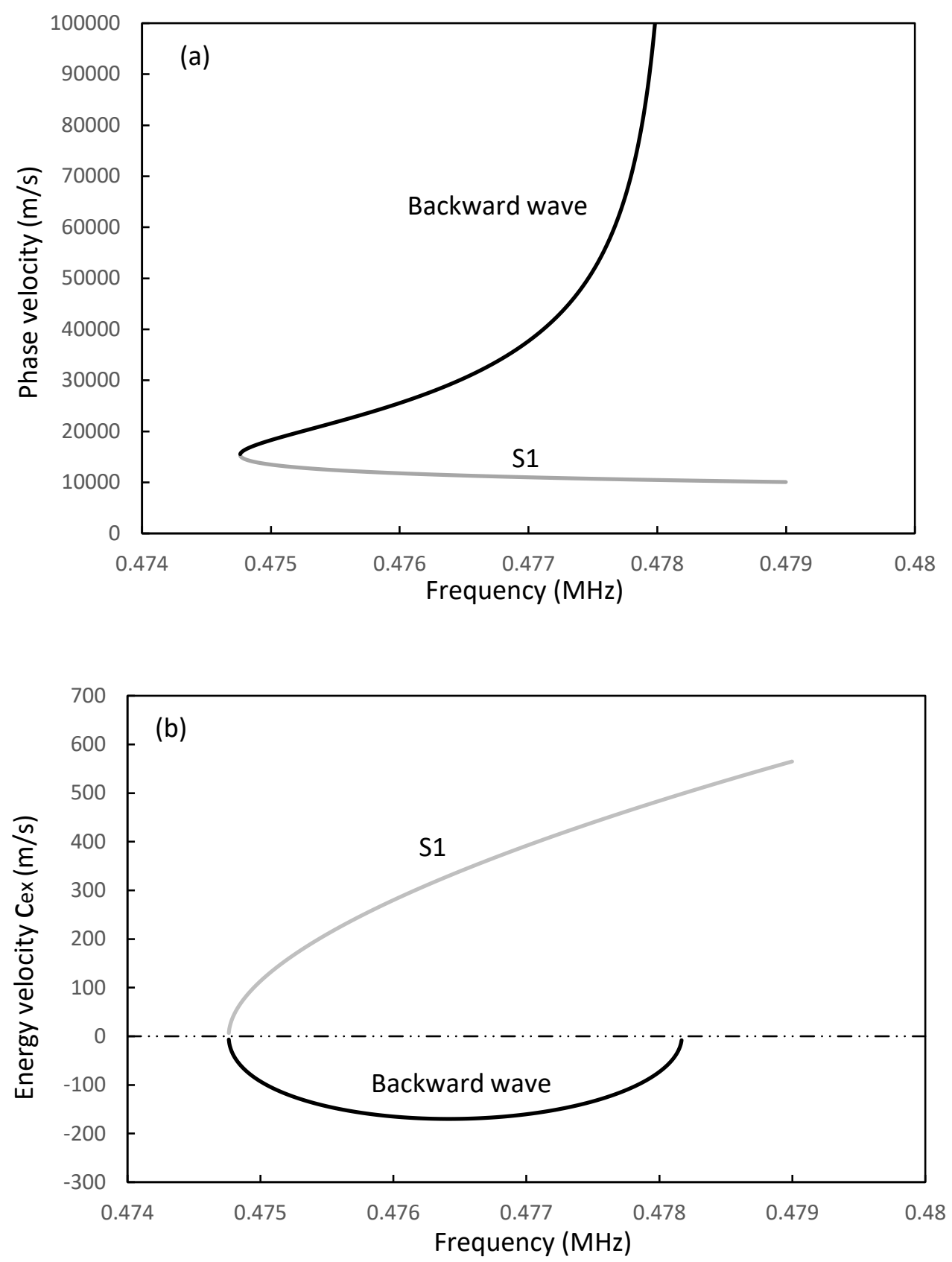


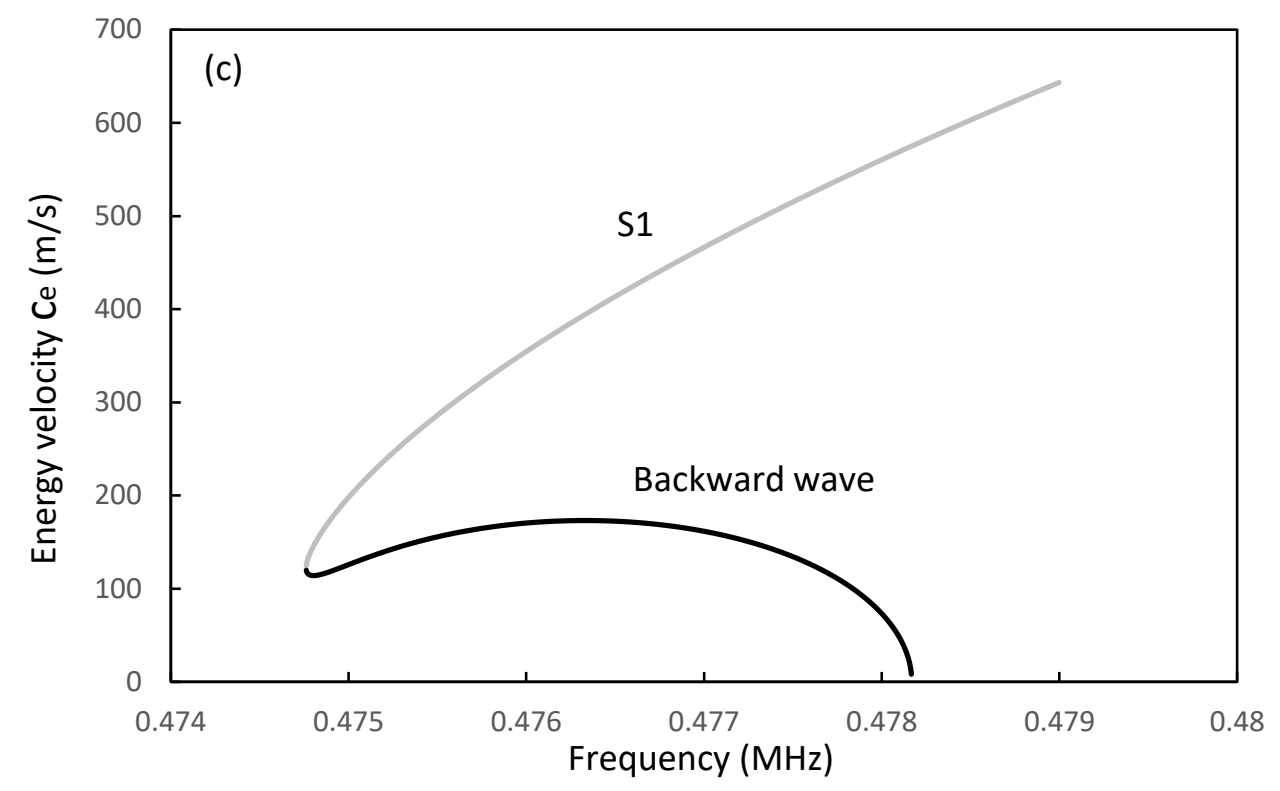

Fig. 6. Dispersion curves for the backward wave propagating alone $30^{\circ}$ direction in the $\left[+45_{6} /-45_{6}\right]_{s}$ laminate: (a) phase velocity; (b) energy velocity component $c_{e x}$; (c) energy velocity $c_{e}$.

To study the existence of the backward wave at all propagation directions, slowness and energy velocity wave front curves are shown in Fig. 7 at a frequency of $0.476 \mathrm{MHz}$. The backward wave has the smallest slowness and energy velocity magnitude, and thus forms the smallest curves in Fig. 7(a) and (b). However, this wave does exist at all the propagation directions, and wave properties are also influenced by fibre orientations. The maximum slowness is pointing in the $45^{\circ}$ direction, and the maximum energy velocity is pointing in the $-45^{\circ}$ direction. These directions are corresponding to fibre orientations in the $\left[+45_{6} /-45_{6}\right]_{s}$ laminate. Note that different to all the other forward waves, the backward wave has a skew angle close to $\pi$ at all propagation directions. The anti-symmetric modes have slowness and energy velocity wave front curves similar to those shown in Figs. 4 and 5, and are thus not shown here for the sake of space. 
(a)

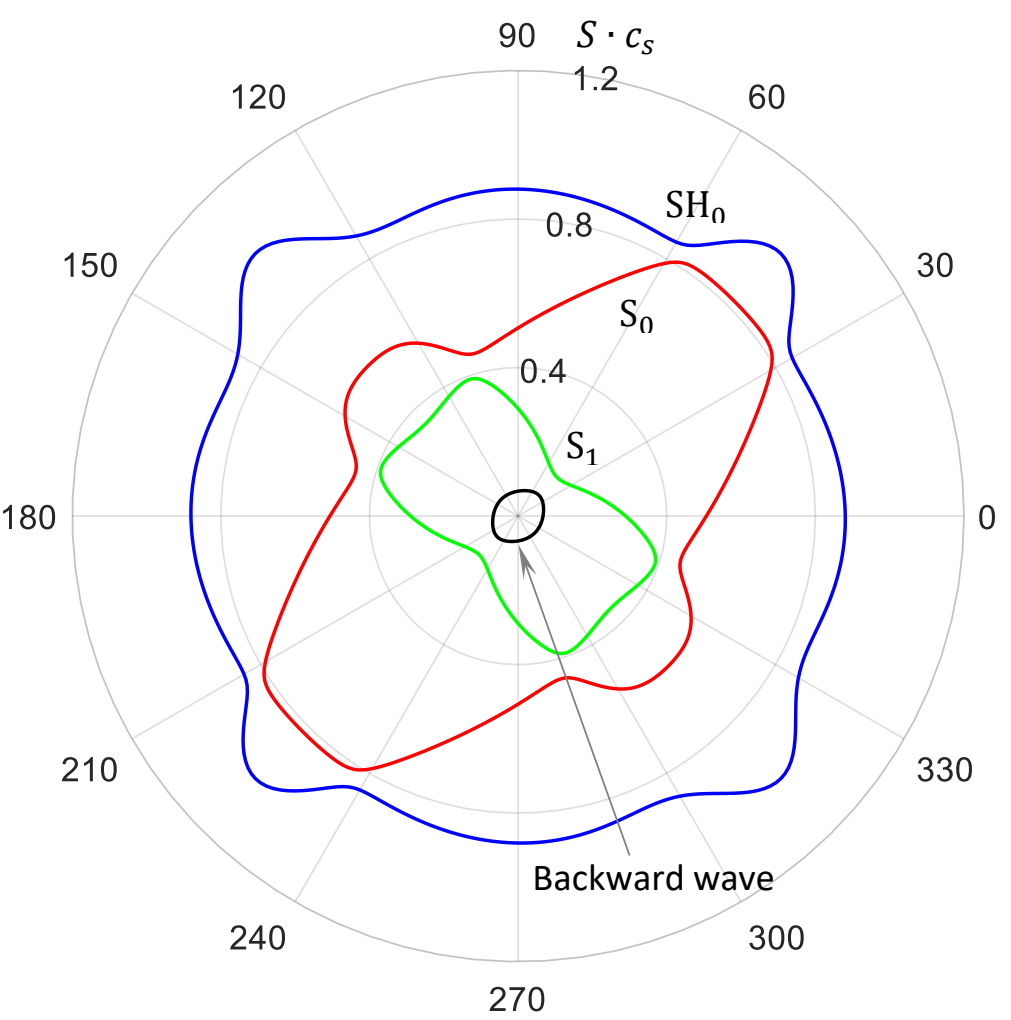

(b)

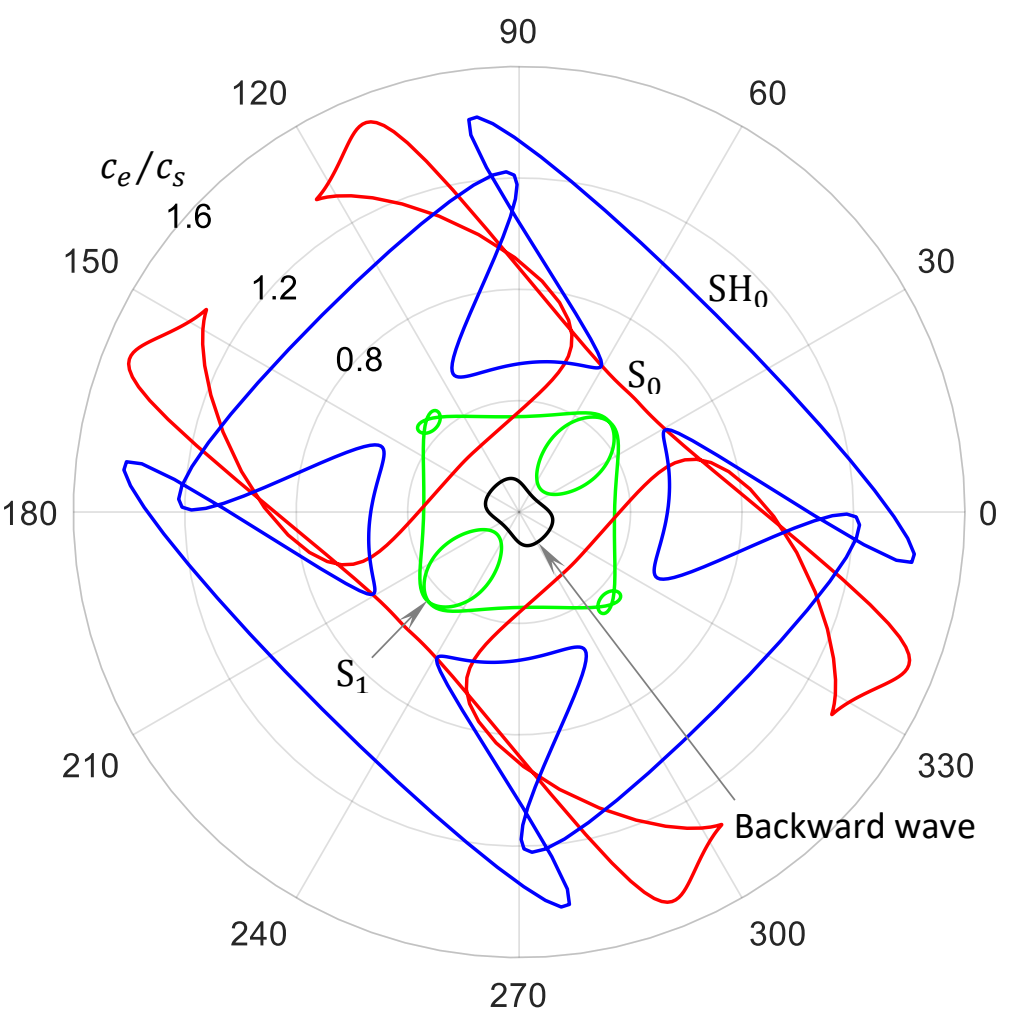


Fig. 7 Angular dependent properties of symmetric and backward waves in the $\left[+45_{6} /-45_{6}\right]_{S}$ laminate at $0.476 \mathrm{MHz}$ : (a) slowness curves; (b) energy velocity wave front curves.

4. Modal characteristics of a non-symmetric laminate

For a symmetric laminate, guided wave modes are either symmetric or anti-symmetric, and these waves could be labelled similar to Lamb and shear-horizontal waves in an isotropic plate. However, for a laminate that is not symmetric with respect to the mid-plane of the laminate, then modes couldn't be exactly symmetric or anti-symmetric. The conventional S and A mode terminologies are thus not used for guided wave modes in these non-symmetric laminates. In this section, a $\left[+45 /-45 / 0 / 90_{2} / 0 /+45 /-45\right]$ laminate is investigated. The total thickness of the laminate is $1 \mathrm{~mm}$. Each ply is made of AS4/3502 with material properties given in Table 1.

Dispersion properties of guided waves propagating alone $30^{\circ}$ direction in the laminate is shown in Fig. 8. At this particular propagation direction, the energy velocity component $c_{e x}$ is almost equal to the total energy velocity $c_{e}$ for all the three fundamental modes in the low frequency range, before the cutting-on frequency of the first high order mode. This laminate is thus different to the $\left[+45_{6} /-45_{6}\right]_{s}$ laminate studied in the previous section, where the fundamental shear-horizontal mode has clear anisotropic material properties even in the low frequency range. However, as frequency increases, $c_{e}$ starts to differ from $c_{e x}$, indicating the existence of the energy velocity component in the direction perpendicular to the wave propagation direction. Fig. 8(b) also shows the existence of a backward wave, with a negative energy velocity in the wave propagation direction. It is thus clear that backward exists in both symmetric and non-symmetric composite materials. 

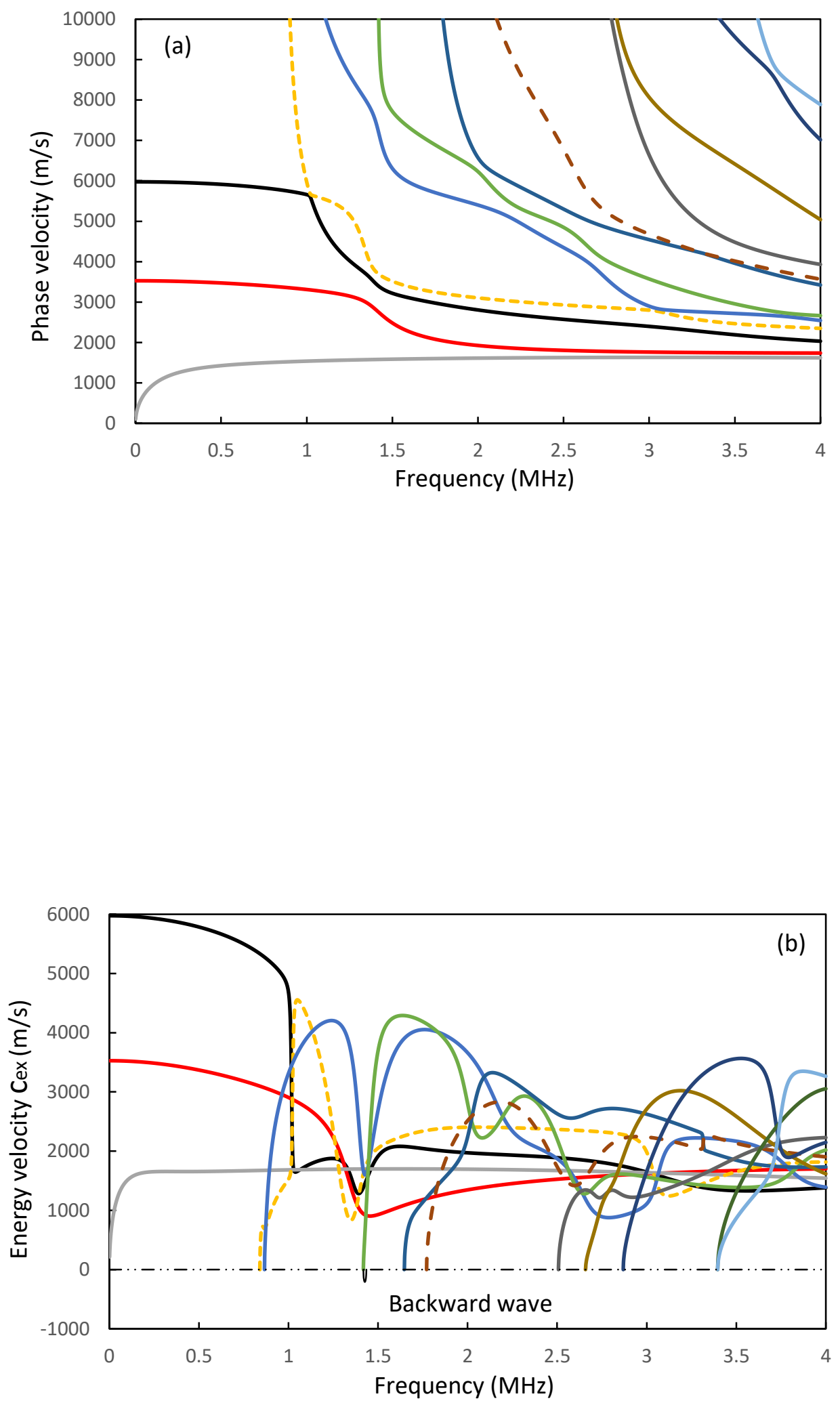


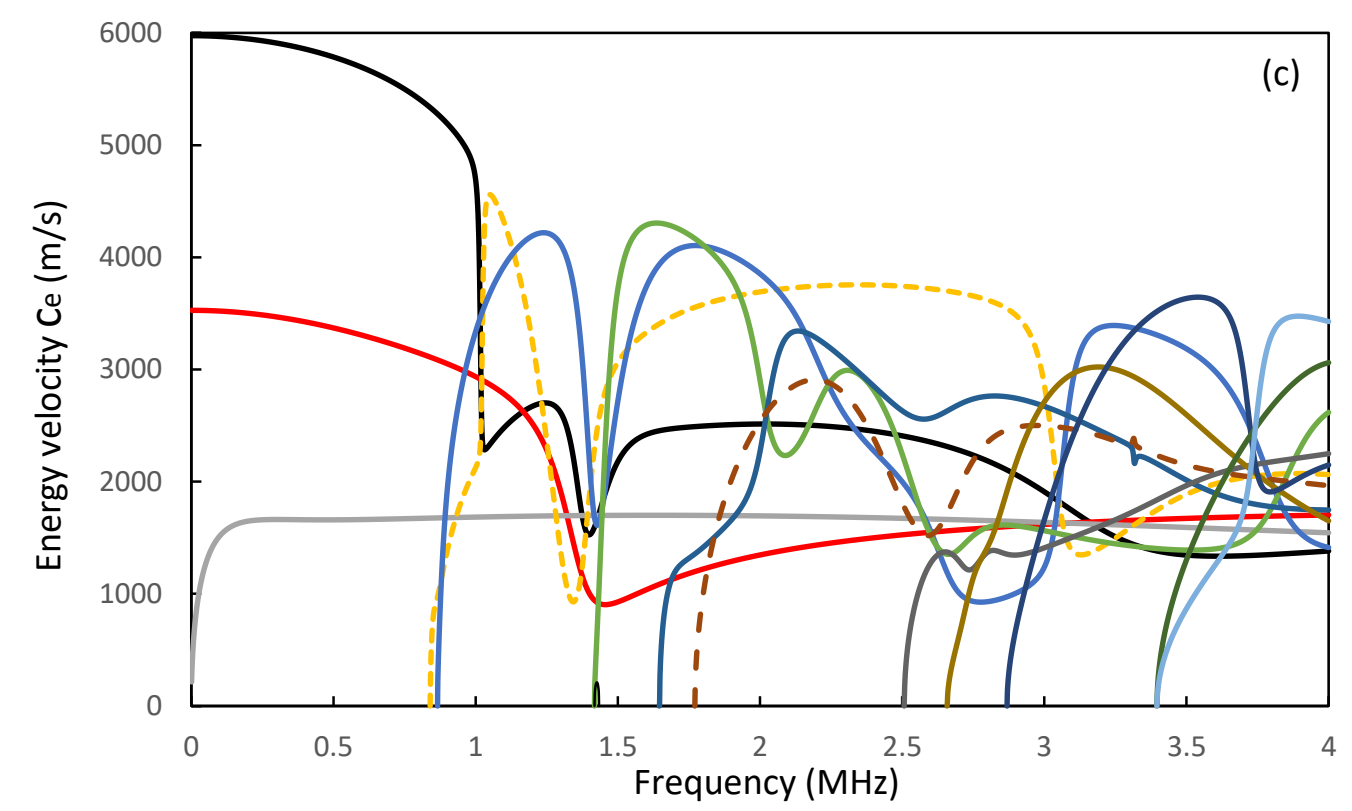

Fig. 8. Dispersion curves for guided waves propagating alone $30^{\circ}$ direction in the [+45/-45/0/90 $2 / 0 /+45 /-45]$ laminate: (a) phase velocity; (b) energy velocity component $c_{e x} ;(\mathrm{c})$ energy velocity $c_{e}$.

To study the angular dependency of modal properties, slowness and energy velocity wave front curves are shown in Fig. 9 at a frequency of $1.42 \mathrm{MHz}$. This frequency is chosen to include the backward wave. At this frequency, one mode has almost constant phase and energy velocities in all directions (the large black circles shown in Figs. 9(a) and (b)). This mode is similar to the fundamental anti-symmetric mode $A_{0}$, studied in the previous section. All the other modes exhibit clear anisotropic material properties. The backward wave has smallest slowness and energy velocity magnitudes, however, this mode does exist at all propagation directions. Fig. 9(b) also shows that most of the modes have multiple energy velocity values at some directions. This indicates that waves propagating in several directions could produce a wave packet overlaid in one direction, thus producing energy focusing. This phenomena is consistent with the modal skew angle profiles shown in Fig. 10. Fig. 10 (a) 
shows that only the quasi $A_{0}$ mode has a skew angle close to zero at all directions. All the other modes have skew angle variations, and this variation could lead to energy focusing. Furthermore, all the forward waves have a skew angle in the vicinity of zero, while the backward wave has a skew angle in the vicinity of $\pi$. This is similar to modal properties in a symmetric laminate.

(a)

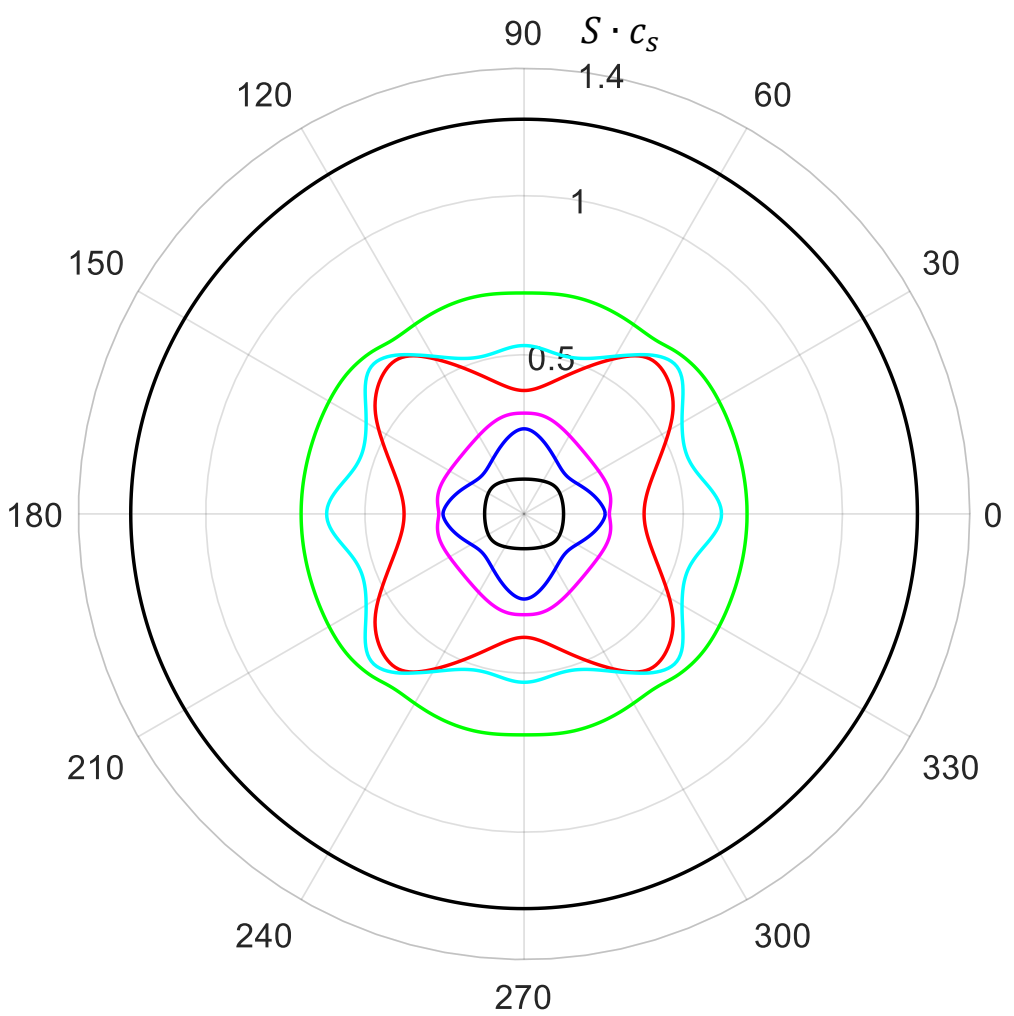


(b)

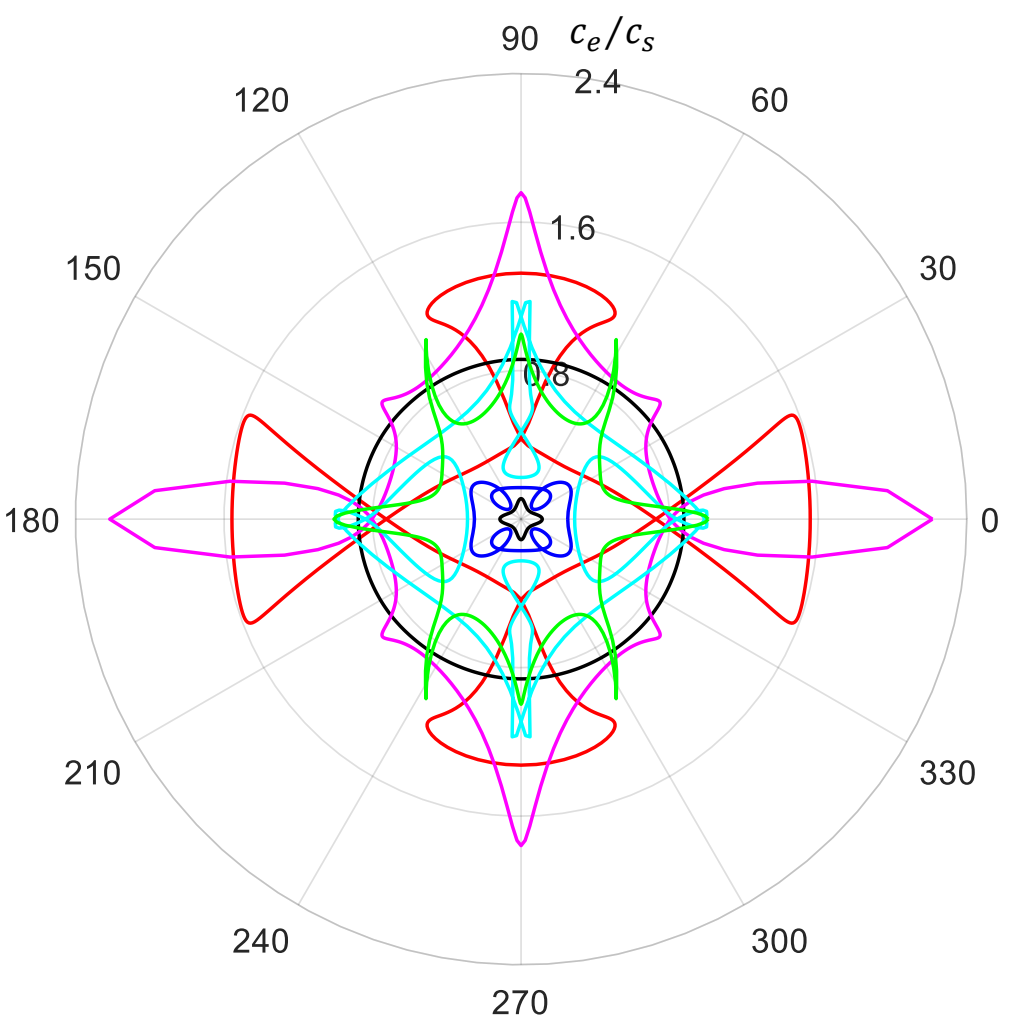

Fig. 9 Angular dependent properties of guided waves in the $\left[+45 /-45 / 0 / 90_{2} / 0 /+45 /\right.$ -45] laminate at 1.42 MHz: (a) slowness curves; (b) energy velocity wave front curves. 
(a)

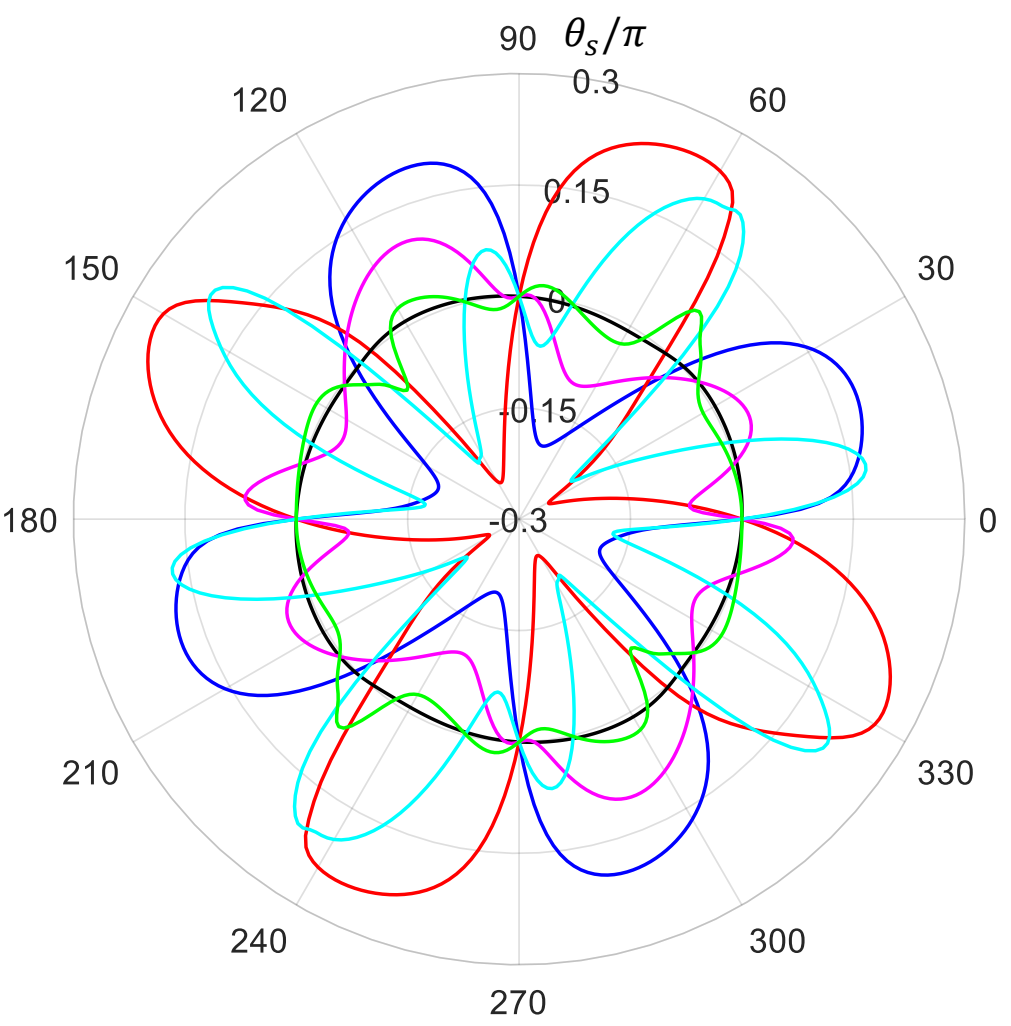

(b)

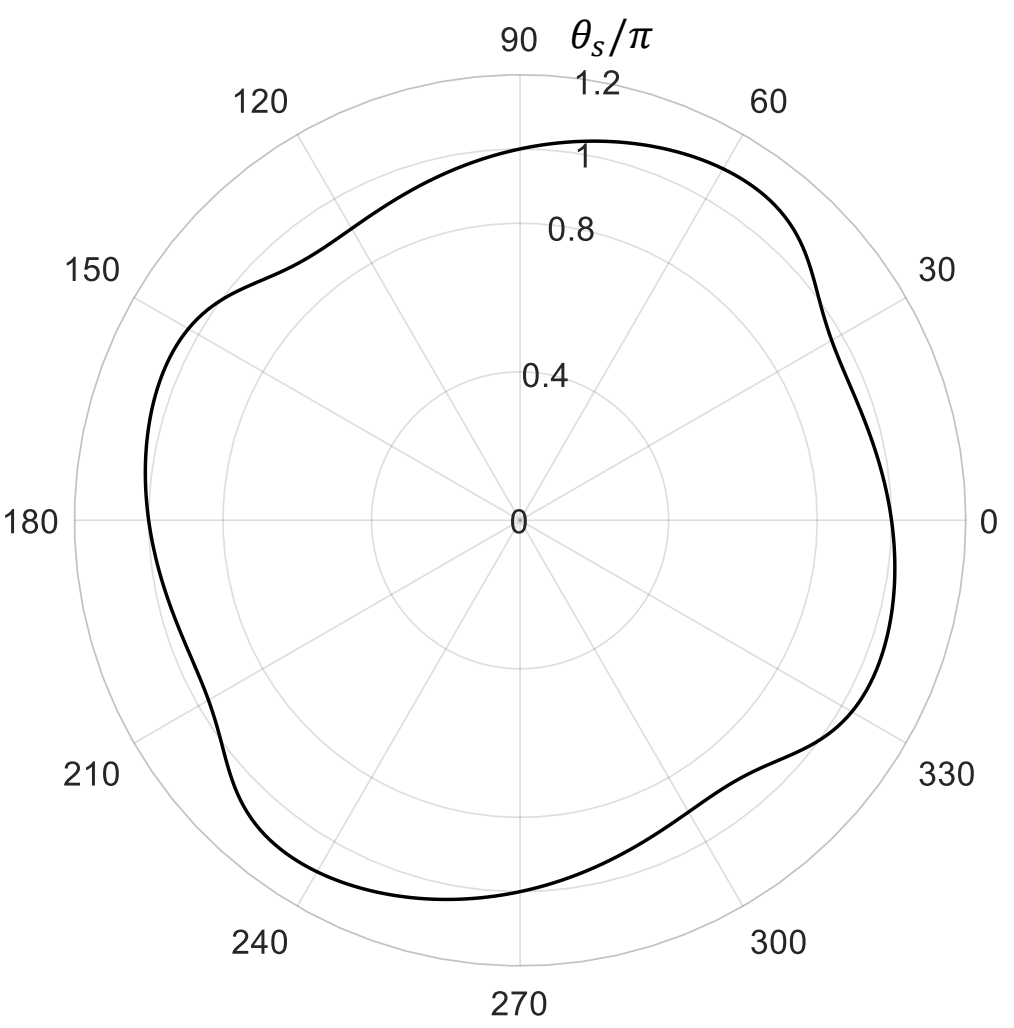


Fig. 10 Skew angles of guided waves in the $\left[+45 /-45 / 0 / 90_{2} / 0 /+45 /-45\right]$ laminate at 1.42 MHz: (a) forward waves; (b) backward wave.

\section{Conclusions}

In this article, a semi-analytical finite element (SAFE) method is developed to study guided wave properties in a composite laminate containing an arbitrary number of layers with an arbitrary fibre orientation in each layer. The weighted residual method is used to derive the weak forms of governing equations. The change of fibre orientation in each layer is incorporated in the model through a coordinate transformation of stiffness constants. The composite layers are then assembled in a global matrix form, and this only requires the thickness of the laminate to be discretised. The eigen equation solution delivers both forward and backward waves. To facilitate the identification of backward waves from forward waves, an energy velocity based formulation is used to calculate energy velocity components in the direction parallel and perpendicular to the wave propagation direction.

The numerical solution is compared to the transfer matrix solution and the Green's matrix solution available in the literature, and excellent agreements have been observed for dispersion curves, slowness and energy velocity wave front curves of forward waves in a AS4/3502 composite laminate. In previous studies, the backward wave has been overlooked, possibly due to the way that the group velocity was calculated and the narrow frequency range of this mode. In this article, it has been shown that backward waves exist in both symmetric and non-symmetric laminates. Furthermore, the backward wave exists in all directions, and the skew angle of the backward wave is in the vicinity of $\pi$. This is different to backward waves in an isotropic plate, where the energy velocity is exactly $180^{\circ}$ out of 
phase with the phase velocity. The angular dependency of forward and backward waves has been examined.

Appendix A: Coordinate transformation

As shown in Fig. $1, x_{j}^{\prime}-y_{j}^{\prime}-z_{j}^{\prime}$ is the local principle coordinate system, and $x-y-z$ is the global coordinate system. The global system could be obtained from a counter-clockwise rotation through an angle $\theta_{j}$ about the $z^{\prime}$ coordinate. Thus, $z$ is identical with $z^{\prime}$, and a clockwise rotation indicates a negative angle. The transformed stiffness constants in the global coordinate system have monoclinic symmetry form:

$$
\begin{gathered}
C_{11}=C_{11}^{\prime} \Upsilon^{4}+C_{22}^{\prime} \Phi^{4}+2\left(C_{12}^{\prime}+2 C_{66}^{\prime}\right) \Phi^{2} \Upsilon^{2} \\
C_{12}=\left(C_{11}^{\prime}+C_{22}^{\prime}-4 C_{66}^{\prime}\right) \Phi^{2} \Upsilon^{2}+C_{12}^{\prime}\left(\Phi^{4}+\Upsilon^{4}\right) \\
C_{13}=C_{13}^{\prime} Y^{2}+C_{23}^{\prime} \Phi^{2} \\
C_{16}=\left(C_{12}^{\prime}-C_{11}^{\prime}+2 C_{66}^{\prime}\right) \Phi Y^{3}+\left(C_{22}^{\prime}-C_{12}^{\prime}-2 C_{66}^{\prime}\right) Y \Phi^{3} \\
C_{22}=C_{11}^{\prime} \Phi^{4}+C_{22}^{\prime} \Upsilon^{4}+2\left(C_{12}^{\prime}+2 C_{66}^{\prime}\right) \Phi^{2} \Upsilon^{2} \\
C_{23}=C_{23}^{\prime} Y^{2}+C_{13}^{\prime} \Phi^{2} \\
C_{26}=\left(C_{12}^{\prime}-C_{11}^{\prime}+2 C_{66}^{\prime}\right) \Upsilon \Phi^{3}+\left(C_{22}^{\prime}-C_{12}^{\prime}-2 C_{66}^{\prime}\right) \Phi \Upsilon^{3} \\
C_{33}=C_{33}^{\prime} \\
C_{45}=\left(C_{44}^{\prime}-C_{55}^{\prime}\right) \Phi \Upsilon \\
C_{44}=C_{44}^{\prime} \Upsilon^{2}+C_{55}^{\prime} \Phi^{2} \\
\left.C_{13}^{\prime}\right) \Phi \Upsilon
\end{gathered}
$$




$$
\begin{gathered}
C_{55}=C_{55}^{\prime} Y^{2}+C_{44}^{\prime} \Phi^{2} \\
C_{66}=C_{66}^{\prime}\left(\Phi^{4}+\Upsilon^{4}\right)+\left(C_{11}^{\prime}+C_{22}^{\prime}-2 C_{12}^{\prime}-2 C_{66}^{\prime}\right) \Phi^{2} \Upsilon^{2}
\end{gathered}
$$

Here, $\Upsilon=\cos \theta_{j}$ and $\Phi=\sin \theta_{j} . C_{q l}^{\prime}(q, l=1,2, \cdots 6)$ are stiffness constants in the local coordinate system, and $C_{q l}(q, l=1,2, \cdots 6)$ are stiffness constants in the global coordinate system.

Appendix B: Local stiffness constants

The stiffness constants in the local principle coordinate system can be expressed in terms of engineering constants as:

$$
\begin{gathered}
C_{11}^{\prime}=\frac{1-v_{23}^{\prime} v_{32}^{\prime}}{E_{2}^{\prime} E_{3}^{\prime} \Delta} \\
C_{22}^{\prime}=\frac{1-v_{13}^{\prime} v_{31}^{\prime}}{E_{1}^{\prime} E_{3}^{\prime} \Delta} \\
C_{12}^{\prime}=\frac{v_{12}^{\prime}+v_{32}^{\prime} v_{13}^{\prime}}{E_{1}^{\prime} E_{3}^{\prime} \Delta} \\
C_{23}^{\prime}=\frac{v_{23}^{\prime}+v_{21}^{\prime} v_{13}^{\prime}}{E_{1}^{\prime} E_{2}^{\prime} \Delta} \\
C_{13}^{\prime}=\frac{v_{13}^{\prime}+v_{12}^{\prime} v_{23}^{\prime}}{E_{1}^{\prime} E_{2}^{\prime} \Delta} \\
C_{33}^{\prime}=\frac{1-v_{12}^{\prime} v_{21}^{\prime}}{E_{1}^{\prime} E_{2}^{\prime} \Delta} \\
C_{44}^{\prime}=G_{23}^{\prime} \\
C_{55}^{\prime}=G_{31}^{\prime} \\
C_{66}^{\prime}=G_{12}^{\prime}
\end{gathered}
$$

where 


$$
\Delta=\frac{1-v_{12}^{\prime} v_{21}^{\prime}-v_{23}^{\prime} v_{32}^{\prime}-v_{31}^{\prime} v_{13}^{\prime}-2 v_{21}^{\prime} v_{32}^{\prime} v_{13}^{\prime}}{E_{1}^{\prime} E_{2}^{\prime} E_{3}^{\prime}}
$$

Furthermore, reciprocal relations must be satisfied:

$$
\frac{v_{q l}^{\prime}}{E_{q}^{\prime}}=\frac{v_{l q}^{\prime}}{E_{l}^{\prime}} \quad q \neq l
$$

References:

1. Lamb H. On Waves in an Elastic Plate. Proc R Soc Lond A 1917; 114-128.

2. Mindlin RD. Mathematical theory of vibrations of elastic plates, in 11th Annual Symposium on Frequency Control. 1957: Asbury Park, NJ, USA, USA. 1-40.

3. Tolstoy I, Usdin E. Wave propagation in elastic plates: low and high mode dispersion. J Acoust Soc Am 1957; 29: 37-42.

4. Negishi K. Existence of negative group velocities in Lamb waves. Jpn J Appl Phys Pt 1 1987; 26: 171-173.

5. Gibson A, Popovics J. Lamb wave basis for impact-echo method analysis. J Eng Mech-ASCE 2005; 131(4): 438-443.

6. Prada C, Balogun O, Murray TW. Laser-based ultrasonic generation and detection of zero-group velocity Lamb waves in thin plates. Appl Phys Lett 2005; 87(19).

7. Clorennec D, Prada C, Royer D. Local and noncontact measurements of bulk acoustic wave velocities in thin isotropic plates and shells using zero group velocity Lamb modes. J Appl Phys 2007; 101(3).

8. Balogun O, Murray T, Prada C. Simulation and measurement of the optical excitation of the S-1 zero group velocity Lamb wave resonance in plates. J Appl Phys 2007; 102(6).

9. Prada C, Clorennec D, Royer D. Local vibration of an elastic plate and zero-group velocity Lamb modes. J Acoust Soc Am 2008; 124(1): 203-212.

10. Clorennec D, Prada C, Royer D. Laser Ultrasonic Inspection of Plates Using ZeroGroup Velocity Lamb Modes. IEEE Trans Ultrason Ferroelectr Freq Control 2010; 57(5): 1125-1132.

11. Ces M, Royer D, Prada C. Characterization of mechanical properties of a hollow cylinder with zero group velocity Lamb modes. J Acoust Soc Am 2012; 132(1): 180185.

12. Tsai Y, Zhu J. Simulation and Experiments of Airborne Zero-Group-Velocity Lamb Waves in Concrete Plate. J Nondestruct Eval 2012; 31(4): 373-382.

13. Grunsteidl C, Veres I, Murray T. Experimental and numerical study of the excitability of zero group velocity Lamb waves by laser-ultrasound. J Acoust Soc Am 2015; 138(1): 242-250.

14. Grunsteidl C, Murray TW, Berer T, Veres IA. Inverse characterization of plates using zero group velocity Lamb modes. Ultrasonics 2016; 65: 1-4. 
15. Bjurstrom H, Ryden N. Detecting the thickness mode frequency in a concrete plate using backward wave propagation. J Acoust Soc Am 2016; 139(2): 649-657.

16. Kausel E. Number and location of zero-group-velocity modes. J Acoust Soc Am 2012; 131(5): 3601-3610.

17. Tofeldt O, Ryden N. Zero-group velocity modes in plates with continuous material variation through the thickness. J Acoust Soc Am 2017; 141(5): 3302-3311.

18. Agounad S, Aassif EH, Khandouch Y, Dcultot D, Maze G, Elhanaoui A. Acoustic scattering from immersed composite cylindrical shells: Existence of zero group velocity circumferential waves. Compos Struct 2017; 182: 12-24.

19. Solie LP, Auld BA. Elastic waves in free anisotropic plates. J Acoust Soc Am 1973; 54(1): 50-65.

20. Nayfeh AH, Chimenti DE. Free wave propagation in plates of general anisotropic media. J Appl Mech-T ASME 1989; 56(4): 881-886.

21. Dayal V, Kinra VK. Leaky Lamb waves in an anisotropic plate. 1. An exact solution and experiments. J Acoust Soc Am 1989; 85(6): 2268-2276.

22. Lin W, Keer LM. A study of Lamb waves in anisotropic plates. J Acoust Soc Am 1992; 92(2): 888-894.

23. Hussain T, Ahmad F. Lamb modes with multiple zero-group velocity points in an orthotropic plate. J Acoust Soc Am 2012; 132(2): 641-645.

24. Laurent J, Royer D, Hussain T, Ahmad F, Prada C. Laser induced zero-group velocity resonances in transversely isotropic cylinder. J Acoust Soc Am 2015; 137(6): 33253334.

25. Shuvalov AL, Poncelet O. On the backward Lamb waves near thickness resonances in anisotropic plates. Int J Solids Struct 2008; 45: 3430-3448.

26. Nayfeh AH, The general problem of elastic wave propagation in multilayered anisotropic media. J Acoust Soc Am 1991; 89(4): 1521-1531.

27. Hosten B, Castaings M. Transfer matrix of multilayered absorbing and anisotropic media. Measurements and simulations of ultrasonic wave propagation through composite materials. J Acoust Soc Am 1993; 94(3): 1488-1495.

28. Potel C, Debelleval JF, Propagation in an anisotropic periodically multilayered medium. J Acoust Soc Am 1993; 93(5): 2669-2677.

29. Castaings M, Hosten B. Delta operator technique to improve the Thomson-Haskell method stability for propagation in multilayered anisotropic absorbing plates. J Acoust Soc Am 1994; 95: 1931-1941.

30. Nayfeh AH. Wave propagation in layered anisotropic media with application to composites. North Holland, 1995.

31. Lowe MJS. Matrix techniques for modeling ultrasonic waves in multilayered media. IEEE Trans Ultrason Ferroelectr Freq Control 1995; 42(4): 525-542.

32. Wang L, Rokhlin S. Stable reformulation of transfer matrix method for wave propagation in layered anisotropic media. Ultrasonics 2001; 39(6): 413-424.

33. Rokhlin S, Wang L. Stable recursive algorithm for elastic wave propagation in layered anisotropic media: Stiffness matrix method. J Acoust Soc Am 2002; 112(3): 822-834.

34. Wang L, Yuan F. Group velocity and characteristic wave curves of Lamb waves in composites: Modeling and experiments. Compos Sci Technol 2007; 67(7-8): 13701384.

35. Karmazin A, Kirillova E, Seemann W, Syromyatnikov P. Investigation of Lamb elastic waves in anisotropic multilayered composites applying the Green's matrix. Ultrasonics 2011; 51: 17-28. 
36. Ma ZY, Chen JL, Li B, Li Z, Su XY. Dispersion analysis of Lamb waves in composite laminates based on reverberation-ray matrix method. Compos. Struct 2016; 136: 419-429

37. Thierry V, Brown L, Chronopoulos D. Multi-scale wave propagation modelling for two-dimensional periodic textile composites. Compos Part B Eng 2018; 150: 144156.

38. Liu T, Karunasena W, Kitipornchai S, Veidt M. The influence of backward wave transmission on quantitative ultrasonic evaluation using Lamb wave propagation. J Acoust Soc Am 2000; 107: 306-314.

39. Gravenkamp H, Song C, Prager J. A numerical approach for the computation of dispersion relations for plate structures using the scaled boundary finite element method, J Sound Vib 2012; 331: 2543-2557

40. Duan W, Kirby R. A numerical model for the scattering of elastic waves from a nonaxisymmetric defect in a pipe. Finite Elem Anal Des 2015; 100: 28-40.

41. Duan W, Kirby R, Mudge P. On the scattering of elastic waves from a nonaxisymmetric defect in a coated pipe. Ultrasonic 2016; 65: 228-241.

42. Duan W, Kirby R, Mudge P, Gan TH. A one dimensional numerical approach for computing the eigenmodes of elastic waves in buried pipelines. J Sound Vib 2016; 384: 177-193.

43. Duan W, Kirby R. Guided wave propagation in buried and immersed fluid-filled pipes: Application of the semi analytic finite element method. Comput Struct 2019; 212: 236-247.

44. Allemang RJ. The modal assurance criterion (MAC): twenty years of use and abuse. In: Proceedings of IMAC 20, the International Modal Analysis Conference. Los Angeles, U.S.A. 2002. 397-405

45. Auld BA. Acoustics Fields and Waves in Solids. Krieger, Malabar FL. 1990. 